\title{
A focal right atrial tachycardia associated with tachycardiomyopathy may mimic a compensatory sinus tachycardia
}

\author{
Philibert $\mathrm{S}^{1 *}$, Laurent $\mathrm{G}^{2,3}$ and Montdesert $\mathrm{B}^{4}$ \\ ${ }^{1}$ European Hospital Georges Pompidou, Paris, France \\ ${ }^{2}$ Cardiology Department, Dijon University Hospital, Dijon, France \\ ${ }^{3}$ University of Burgundy, Dijon, France \\ ${ }^{4}$ Montreal Heart Institute, Canada
}

\begin{abstract}
We report a case of a 19-year old man with incessant right atrial tachycardia (AT) resulting in severe biventricular systolic dysfunction. Successful catheter ablation was obtained by cryo-energy applications at two right atrial exits and it was followed by full recovery of ventricular contractility at 3 months evaluation.
\end{abstract}

\section{Introduction}

Tachycardia-induced cardiomyopathy (TIC), which was first reported in 1986 by Packer et al. [1] is a reversible myocardial injury that is the result of sustained tachyarrhythmia. Abnormal atrial, junctional or even ventricular rhythms may be responsible for TIC, but very little is known about TIC as a result of focal atrial tachycardia (AT).

\section{Case presentation}

A 19-year-old man was admitted to our center with worsening dyspnoea and tachycardia for several months. He complained of paroxysmal palpitations (about $120 \mathrm{bpm}$ ) at rest without any changes in blood pressure or respiratory distress. The physical examination was normal, with no symptoms of heart murmur or heart failure. There was no family history of cardiac disease or sudden cardiac death.

On examination, the ECG showed an AT at $120 \mathrm{bpm}$ with a narrow QRS complex (96 ms), a normal QRS axis and QT interval, biphasic $\mathrm{P}$ waves in the inferior leads (with a first negative component), and negative $\mathrm{P}$ waves in aVR (Figure 1A). Trans-thoracic echocardiogram (TTE) revealed a left ventricular ejection fraction (LVEF) of $30 \%$ without any left ventricular (LV) dilation (Left ventricular end diastolic diameter (LVEDD) in parasternal long axis view: $48 \mathrm{~mm}$ ). Tests for thyroid function, NT-proBNP, electrolytes, troponin and D-dimers were normal. Cardiac magnetic resonance imaging (MRI) confirmed severe bi-ventricular dysfunction without dilation of $28 \%$ and $84 \mathrm{ml} . \mathrm{m}^{-2}$ for the $\mathrm{LV}$ and $32 \%$ and $66 \mathrm{ml} \cdot \mathrm{m}^{-2}$ for the right ventricle, respectively, without myocardial delayed enhancement. Direct intravenous adenosine infusion (12 mg) temporarily stopped the AT (therefore confirming the diagnosis) with a single beat in sinus rhythm before AT resumed (Figure 1B). Cardio-selective beta-blockers (Metoprolol 25 $\mathrm{mg}$ BID) did not result in rhythm control or even manage to slow down the tachycardia.

After patient consent was obtained, an invasive electrophysiological (EP) study was performed. The cycle length of the tachycardia was
450-460 ms with predominant negativity of the $\mathrm{P}$ waves in the inferior leads (Figure 2) as opposed to SR with positive P waves in lead II and aVF (540ms CL). The electroanatomic mapping with the NAV'X 3D Velocity ${ }^{\circledR}$ System (St. Jude Medical, St. Paul, Minneapolis, USA) showed that the earliest atrial activation during AT was in the high right atrium (RA) below the sinus node and close to the tricuspid annulus (Figure 3). For a question of catheter stability in this region, we decided to use cryo-energy (Cryocatheter Freezor ${ }^{\circledR} 6 \mathrm{~mm}$ blue curve, Medtronic, Minneapolis, USA). Intravenous adenosine was administered to temporarily restore the SR in order to distinguish AT from SR when mapping the atrial activation sequence in the RA. We recorded changes in activation along the decapolar catheter placed around the tricuspid annulus (distal RA 1-2 just below the base of the right atrial appendage, and proximal RA 9-10 at the right atrial roof during AT vs SR, whereas the coronary sinus (CS) activation sequence was unchanged (Concentric from proximal CS 9-10 to distal CS 1-2)) (Figure 2). The exact location of the phrenic nerve was identified and tagged with the ablation catheter using high voltage stimulation. A cryo-application at the earliest atrial activation (Figure 2) resulted in a slowdown followed by an abrupt stop of the AT. We completed two additional applications of $240 \mathrm{~s}$ each and were still able to re-induce AT on isoproterenol $2 \mathrm{mg} / \mathrm{h}$ with burst pacing. We then re-mapped activation on top of the original results (Figure $3 \mathrm{~A}$ ), which is why the map displays different white tagged areas. Below the first exit site we found a second exit site which was even closer to the tricuspid annulus. A new set of applications provided an immediate effect: AT was no longer inducible despite atrial bursts on isoproterenol infusion $(2 \mathrm{mg} / \mathrm{h})$. TTE and MRI performed 3 months after ablation showed normalized right and left ventricular function.

${ }^{*}$ Correspondence to: Philibert S, European Hospital Georges Pompidou, Paris, France, E-mail: severine.philibert-ext@aphp.fr

Received: August 05, 2019; Accepted: August 23, 2019; Published: August 26 , 2019 
Philibert S (2019) A focal right atrial tachycardia associated with tachycardiomyopathy may mimic a compensatory sinus tachycardia

A

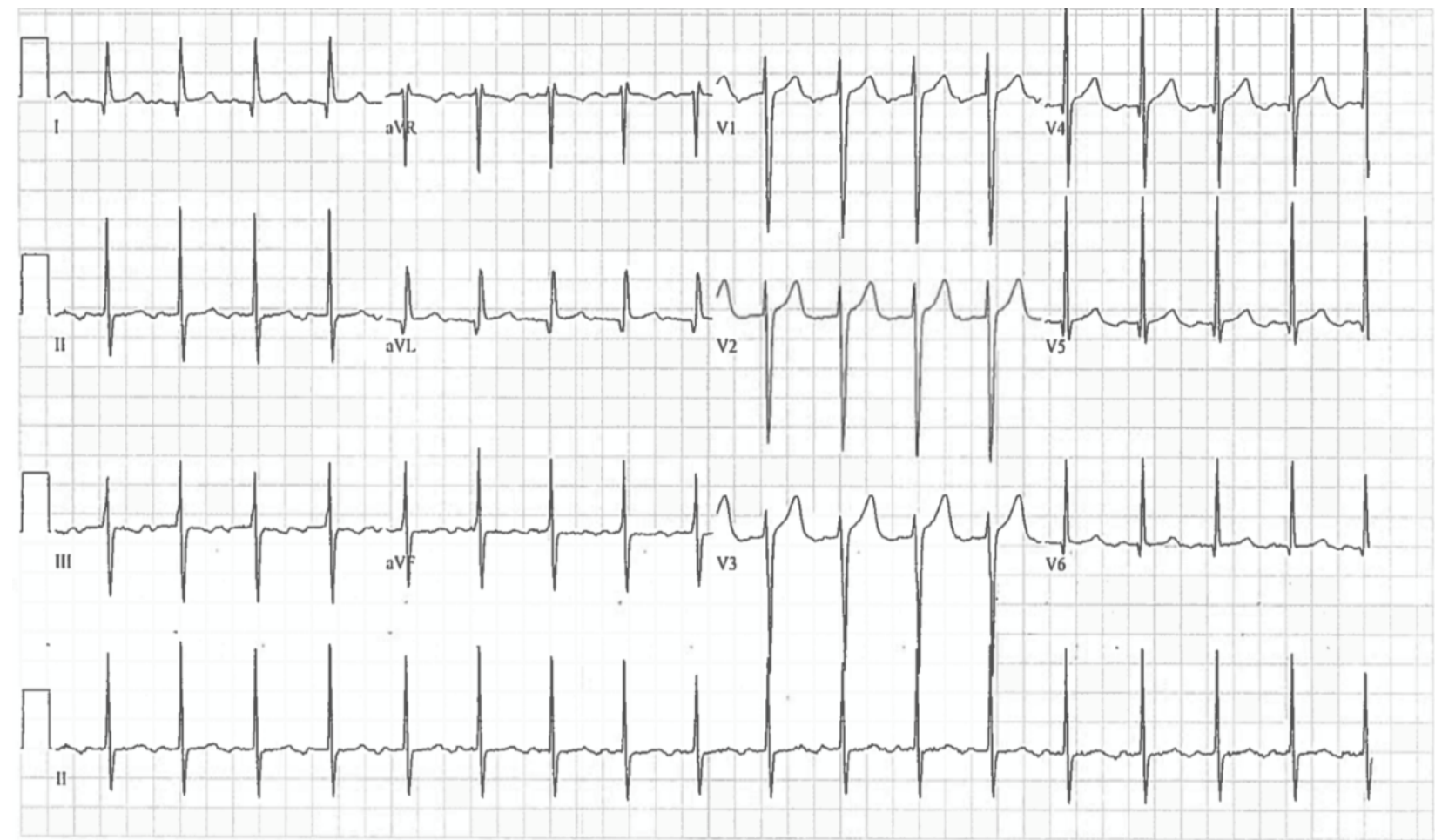

$25 \mathrm{~mm} / \mathrm{s} \quad 10 \mathrm{~mm} / \mathrm{mV} \quad 100 \mathrm{~Hz} \quad 9.0 .0 \quad 12 \mathrm{SL} 239 \quad$ IDC: 62

SID: 8963255 EID:4893 EDT: 11:16 08-fév-2016 ORDRE:

B

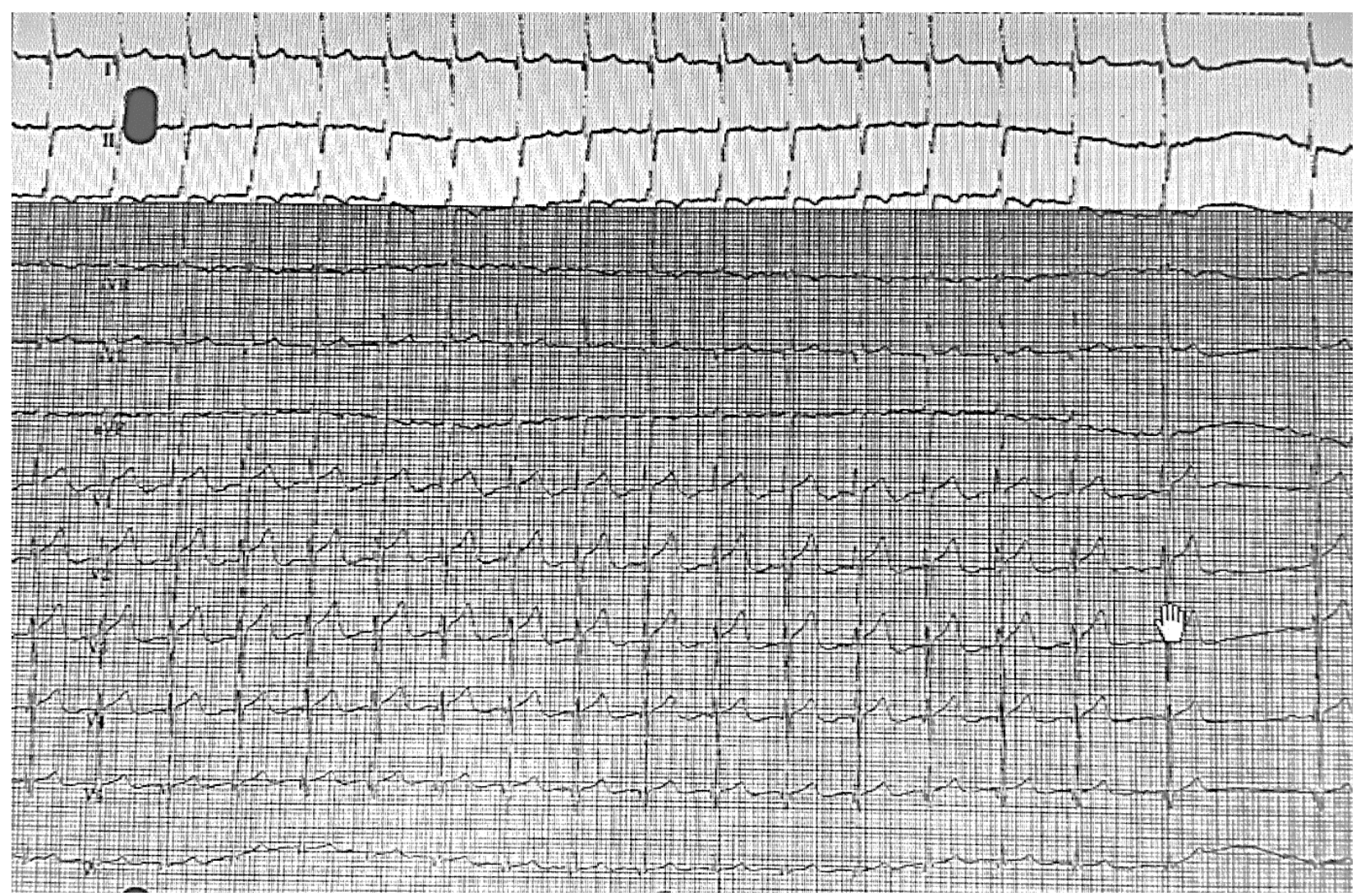

Figure 1. 12 lead-surface ECG recorded in the emergency room A) Narrow complex tachycardia at 120 beats per minute with negative P wave in inferior leads. B) Adenosine testing during atrial tachycardia: the first beat in sinus rhythm (before AT resumed) shows a variation in P wave morphology (positive in inferior leads) 


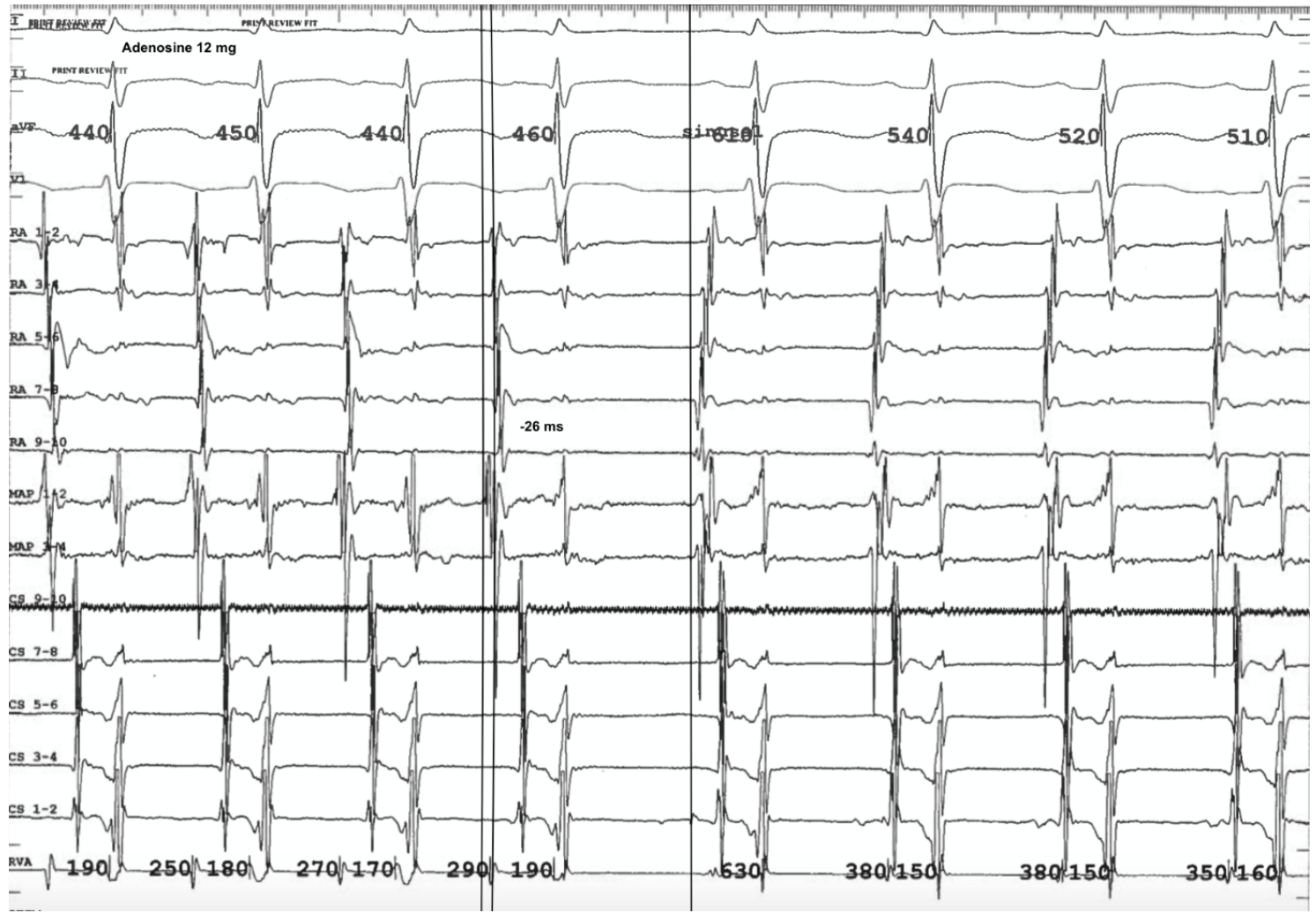

Figure 2. Electrophysiological study at $100 \mathrm{~mm} / \mathrm{sec}$ wipe speed:

- Surface ECG lead I, II, aVF and V1

- Right atrium (RA) EGMs were recorded with a decapolar catheter placed around the tricuspid annulus, distal RA 1-2 just below the RAA foot, and proximal RA 9-10 at the roof of the RA

- MAP is the mapping catheter used with the 3-D navigation system.

- Coronary sinus (CS) EGMs were recorded with a decapolar catheter placed in the CS (CS 1-2 for distal to CS 9-10 for proximal CS)

- Right ventricular (RV) EGM was recorded with a bipolar catheter placed at the RV apex (RVA)

Left hand side of the tracing: Surface ECG and intra-cardiac EGMs demonstrated a stable narrow-QRS tachycardia with a 450-460 m sec cycle length (CL) and with a negative P wave in inferior leads. The first atrial activation in tachycardia is recorded at MAP 1-2 preceding RA 1-2 by $26 \mathrm{~ms}$. Right hand side of the tracing: Sinus rhythm is restored after IV Adenosin, and we can see a positive $\mathrm{P}$ wave in the inferior leads. There are changes in the atrial activation sequence along the RA catheter (first activation at RA 7-8), whereas there are no changes in CS activation (concentric). MAP 1-2 EGM, success site during ablation, was recorded simultaneously with the first atrial activation on RA catheter in sinus rhythm. Note that atrial and ventricular EGMs are of similar amplitudes at the AT site of origin or during sinus rhythm indicating a peri-annular position.
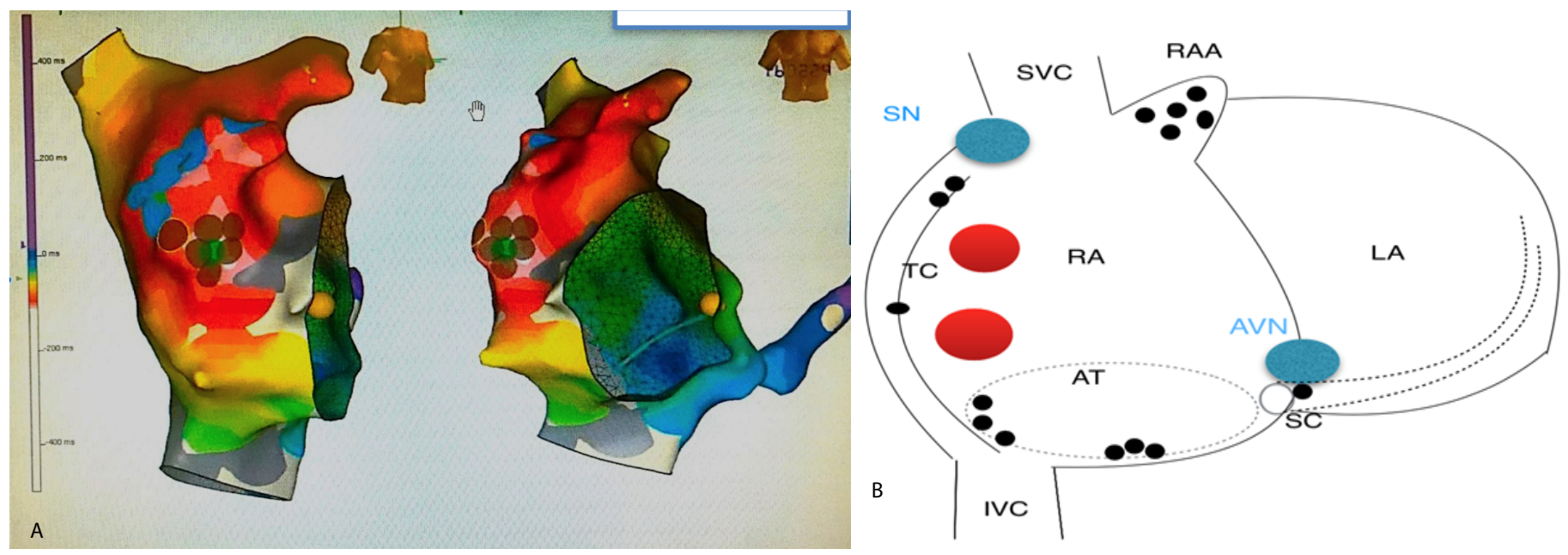

Figure 3A. AP and LAO views of the 3-D activation map during atrial tachycardia using Nav’X Velocity ${ }^{\circledR}$ system (St. Jude Medical, St. Paul, Minnesota, USA). In the first activation map, the earliest atrial activation (areas tagged in white) was initially located just below the RAA and therefore the sinus node. Several cryoapplications were done at this site, resulting in a transient effect on AT (Blue dots). A second mapping sequence was performed on top of the first while AT resumed. A new exit site was located below the first ablation site on the lateral wall of the RA (new set of white tags). Ablation at the second identified site was permanently successful (brown dots). B) AP view of a scheme of the right atrium showing the usual locations of the sinus and AV nodes (blue dots), and the two areas from which AT originated (red dots) and from which they were subsequently ablated in this case. The black dots indicate where AT is typically located in the right atrium. 


\section{Discussion}

We reported a rare case of a permanent peri-annular TIC which did not respond to medication and was successfully treated by cryoablation in a young adult. Cardiac function was already improved 72 hours after ablation, and completely restored at 3 months. Even if AT is considered rare, Weizhu Ju et al. [2] found that $8.3 \%$ of patients with focal AT suffered from TIC. Younger age (22 years old or less), persistent arrhythmia and tachycardia were independent risk factors for developing TIC [2-3].

It may be challenging to diagnose a focal AT from surface ECG because it can mimic sinus tachycardia. This is especially true when the rate is not particularly fast and if the site of origin is close to the sinus node. In our case, the tachycardia was previously considered a sinus tachycardia with a "suitable" compensatory response to LV dysfunction. Unremitting paroxysmal AT with a relatively slow rate may easily be responsible for TIC because the tachycardia remains undiagnosed and mostly asymptomatic.

A detailed P wave morphology analysis on 12-lead surface ECG can help to rule out sinus tachycardia. Kistler et al. have described an algorithm for detailed $\mathrm{P}$ wave morphology (PWM) analysis that can predict the original anatomical site of any AT with 93\% accuracy. During tachycardia, we observed slight cycle length variations (440$460 \mathrm{~ms}$ ) suggesting an automatic mechanism. The focal origin and sensitivity to adenosine confirmed this hypothesis. Automatic foci are usually found in adults along the crista terminalis, the tricuspid annulus, around the coronary sinus ostium or in the perinodal region, whereas the most common location in children is the right atrial appendage (Figure 3B) [4]. In adults, adenosine-sensitive AT is mainly found in the perinodal area or around the valvular rings. In our case, two sets of cryo-applications were needed to eradicate the AT which is unusual. It could be either because the AT had dual exit sites or that the first set of applications (close to the actual site of origin) transiently inactivated part of the AT substrate.

\section{Conclusion}

We report here a case of a young man with tachycardia-induced cardiomyopathy in whom permanent peri-annular atrial tachycardia was mistaken for compensatory sinus tachycardia. Careful $\mathrm{P}$ wave morphology analysis on surface ECG made the early identification of this disorder possible. Appropriate treatment stopped the progression of left ventricular dysfunction, and function was completely recovered after successful cryoablation of the focal site of origin without any further complications.

\section{References}

1. Packer DL, Bardy GH, Worley SJ, Smith MS, Cobb FR, et al. (1986) Tachycardiainduced cardiomyopathy: a reversible form of left ventricular dysfunction. $\mathrm{Am} \mathrm{J}$ Cardiol 57: 563-570. [Crossref]

2. Weizhu J, Bing Y, Minglong C, Zhang F, Chen H, et al. (2014) Tachycardiomyopathy Complicated by Focal Atrial Tachycardia: Incidence, Risk Factors, and Long-Term Outcome. J Cardiovasc Electrophysiol 25: 953-957. [Crossref]

3. Medi C, Kalman J, Haqqani H, Vohra JK, Morton JB, et al. (2009) TachycardiaMediated Cardiomyopathy Secondary to Focal Atrial Tachycardia. J Am Coll Cardiol 53: 1791-1797. [Crossref]

4. Kistler PM, Fynn SP, Haqqani H, Stevenson IH, Vohra JK, et al. (2005) Focal atrial tachycardia from the ostium of the coronary sinus: electrocardiographic and electrophysiological characterization and radiofrequency ablation. J Am Coll Cardiol 45: 1488-1493. [Crossref]

Copyright: (C2019 Philibert S. This is an open-access article distributed under the terms of the Creative Commons Attribution License, which permits unrestricted use, distribution, and reproduction in any medium, provided the original author and source are credited. 